\title{
Switchable Plasmonic Metasurfaces with High Chromaticity Containing Only Abundant Metals
}

Kunli Xiong, Daniel Tordera, Gustav Emilsson, Oliver Olsson, Ulrika Linderhed, Magnus P J onsson and Andreas B Dahlin

The self-archived postprint version of this journal article is available at Linköping University Institutional Repository (DiVA):

http:// urn.kb.se/ resolve?urn=urn:nbn:se:liu:diva-143686

N.B.: When citing this work, cite the original publication.

Xiong, K., Tordera, D., Emilsson, G., Olsson, O., Linderhed, U., J onsson, M. P, Dahlin, A. B, (2017), Switchable Plasmonic Metasurfaces with High Chromaticity Containing Only Abundant Metals, Nano letters (Print), 17(11), 7033-7039. https:// doi.org/ 10.1021/ acs.nanolett.7b03665

Original publication available at:

https:// doi.org/ 10.1021/acs.nanolett.7b03665

Copyright: American Chemical Society

http:// pubs.acs.org/ 


\section{Switchable Plasmonic Metasurfaces with High Chromaticity Containing Only Abundant Metals}

Kunli Xiong, ${ }^{1}$ Daniel Tordera, ${ }^{2}$ Gustav Emilsson, ${ }^{1}$ Oliver Olsson, ${ }^{3}$ Ulrika Linderhed, ${ }^{4}$ Magnus. P. Jonsson ${ }^{2}$ and Andreas B. Dahlin. ${ }^{1 *}$

1 Dept. of Chemistry and Chemical Engineering, Chalmers University of Technology, 41296 Göteborg, Sweden.

2 Linköping University, Dept. of Science and Technology, Laboratory for Organic Electronics, 60174 Norrköping, Sweden.

3 rdot AB (559092-9831), Stena Center 1, 41292 Göteborg, Sweden.

4 RISE Acreo, Box 787, 60117 Norrköping, Sweden. 


\begin{abstract}
Plasmonic color generation offers several advantages but is also limited by the cost and availability of noble metals like gold. In this work we present color-tuneable metasurfaces with high chromaticity and reflectivity consisting of an aluminum mirror, a dielectric spacer and a plasmonic nanohole array in copper. Copper is shown to be an excellent alternative to gold when properly protected from oxidation and makes it possible to generate a wide RGB gamut covering $27 \%$ of the standard RGB. By patterning the metasurfaces into microscale pixel triplets, color photos can be well reproduced with high resolution over wafer-sized areas. Further, we demonstrate active modulation of the reflected intensity using an electrochromic conductive polymer deposited on top of the nanostructures by screen printing. This technology opens up for ultrathin and flexible reflective displays in full color, i.e. plasmonic electronic paper, compatible with large-scale sustainable production.
\end{abstract}

KEYWORDS: plasmons, colors, nanostructures, electrochromism, electronic paper

\title{
TOC graphic
}


Plasmonic nanostructures have been used to generate decorative colors for thousands of years. ${ }^{1}$ Recently there has been a renowned interest in utilizing such strong light-matter interactions for color generation ${ }^{2,3}$ and active electrochromic devices. ${ }^{4,5}$ Some of the most prominent advantages of plasmonic coloration are that ultrathin nanostructures or "metasurfaces" are sufficient for high chromaticity and that the structures are generally more robust than organic dyes. Also, by nanofabrication techniques, plasmonic images can be produced at extremely high resolution. ${ }^{6-8}$ Furthermore, various techniques can be implemented in order to make the picture display dynamic. ${ }^{9}$ If a conductive polymer is grown on the plasmonic surface, electrochromic switching becomes possible and the colors are clearer than those generated by polymers alone. ${ }^{10}$ Using suitable nanostructures this technology opens up for reflective displays operating in color. ${ }^{11}$

However, plasmonic devices are generally based on noble metals (mainly Au) which are rare and expensive. This limits potential applications and production of functional devices. Although little material is needed for ultrathin nanostructures in principle, much is lost in the fabrication process, especially since vacuum deposition techniques are almost always necessary. This has lately led to an intense focus on "sustainable plasmonics", i.e. the use of abundant metals, in particular aluminum ${ }^{12}(\mathrm{Al})$. Still, color generation with nanostructures based on $\mathrm{Al}$ has several limitations to date. The structures are often only prepared on small areas because of the serial nature of the nanolithography techniques used ${ }^{13,14}$ and although the color range can be impressive considering that only one metal is used, ${ }^{15-17}$ there is still room to improve the chromaticity. Also, many devices have been designed for dark-field illumination ${ }^{13}$ (light scattering), but this configuration is more suitable for sensing applications ${ }^{18}$ rather than electrochromic devices. Further, the only implementation of active intensity modulation is liquid crystals above the nanostructures, ${ }^{4}$ which limits the overall reflectivity/transmittance due to the polarization 
dependence and makes the pixels considerably thicker. This in turn makes the system unbendable as well as sensitive to the viewing angle. Naturally, many of these limitations apply also when noble metals are used for plasmonic color generation and in electrochromic devices.

In this work we present high chromaticity plasmonic metasurfaces based on a metal - insulator - nanohole array (MIN) thin film configuration containing only abundant metals. We evaluate the relatively unexplored ${ }^{19}$ metal copper $(\mathrm{Cu})$ for plasmonic color generation and show that this can generate a much wider gamut compared to $\mathrm{Al}$ alone. The MIN structures show high reflectivity and can be patterned over wafer sized areas. Colorful pictures can be clearly reproduced at high resolution using red, green and blue pixel triplets. In addition, we show that an electrochromic polymer film can be prepared on the MIN structures by screen printing, which enables on/off switching of the colors without the need for polarizers. In simple prototypes we demonstrate that this technology can provide flexible reflective displays with ultralow power consumption and compatibility with large scale production due to solely cheap materials.

Motivated by the low price of $\mathrm{Cu}$ (comparable to $\mathrm{Al}$ ) we started by characterizing the optical response of semitransparent $\mathrm{Cu}$ films containing short-range ordered nanoholes prepared by colloidal lithography. ${ }^{20-23}$ Plasmonic activity in $\mathrm{Cu}$ is relatively unexplored in the literature ${ }^{24}$ because spontaneous oxidation normally causes too high damping, ${ }^{25}$ although chemical modifications may provide stability at least for the timescale of the experiments. ${ }^{26}$ Nanoholes in $\mathrm{Cu}$ have been prepared previously ${ }^{27}$ but their optical response has to the best of our knowledge never been characterized, most likely due to the problem of oxidation. We protected the metal by an additional $20 \mathrm{~nm}$ alumina $\left(\mathrm{Al}_{2} \mathrm{O}_{3}\right)$ coating (Fig. 1A) deposited in the same electron gun evaporator run, such that the $\mathrm{Cu}$ film is never exposed to air in between. This protection provided excellent long-term stability (in air) as verified by no significant changes in the optical properties 
over the timescale of the study ( 1 year). Due to the relatively similar permittivity of $\mathrm{Au}$ and $\mathrm{Cu}$ (when free from oxidation), ${ }^{28}$ we expected the far field spectra to be comparable to structurally identical short-range ordered nanohole arrays prepared in semitransparent Au films (see literature). ${ }^{20-23}$ Indeed, the extinction spectra of nanoholes in a $30 \mathrm{~nm} \mathrm{Cu}$ films (standard error \pm 1 $\mathrm{nm}$ ) showed the characteristic peak in the red, representing coupling to surface plasmons by the short-range ordering of the apertures (Fig. 1B), ${ }^{20}$ followed by an enhanced transmission at nearby longer wavelengths (Fig. 1C). The resonance features are only slightly broadened compared to Au nanohole arrays. ${ }^{20-23}$ This can to some extent be attributed to the fact that in contrast to Au there is no need for an adhesion layer ( $\mathrm{Ti}$ or $\mathrm{Cr}$ ) under the $\mathrm{Cu}$ film on glass. Note that the colloidal lithography provides a saturated monolayer and that the number density of holes should not be reduced since it destroys the short-range ordering and consequently the plasmonic activity. ${ }^{20}$ The resonances redshift with increasing the hole (colloid) diameter, which is due to the increased effective periodicity of the array. The hole diameter itself does not influence the resonance wavelength although larger holes give more scattering. ${ }^{23}$ Furthermore, the resonances also redshift as the film thickness decreases to $20 \mathrm{~nm}$ (Fig. 1D) as predicted by the dispersion relation of the bonding surface plasmon mode in the finite film. ${ }^{20,29}$ 


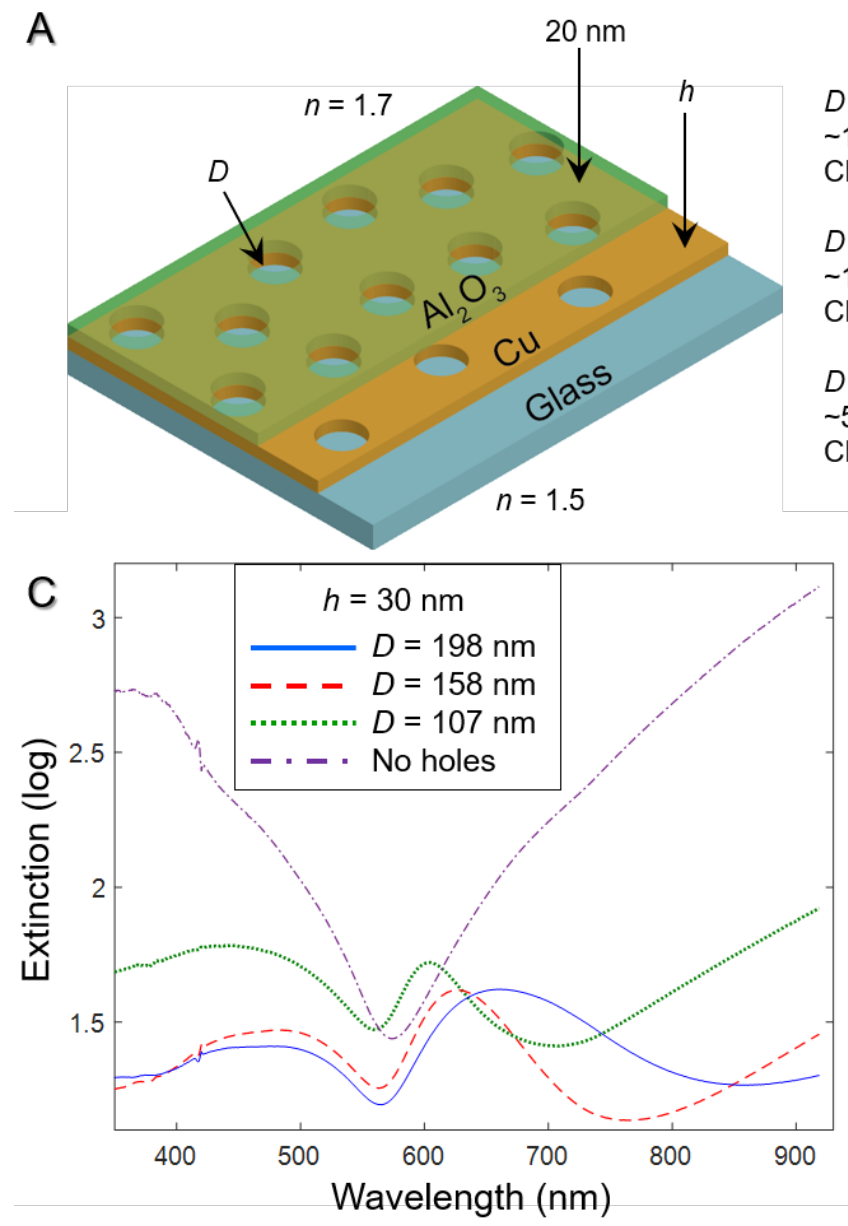

$D=107 \mathrm{~nm} \rightarrow$

Characteristic spacing $\sim 210 \mathrm{~nm}$

$D=158 \mathrm{~nm} \rightarrow$

$\sim 10$ holes $/ \mu \mathrm{m}^{2}$

Characteristic spacing $\sim 290 \mathrm{~nm}$

$D=198 \mathrm{~nm} \rightarrow$

$\sim 5$ holes $/ \mu \mathrm{m}^{2}$

Characteristic spacing $\sim 390 \mathrm{~nm}$
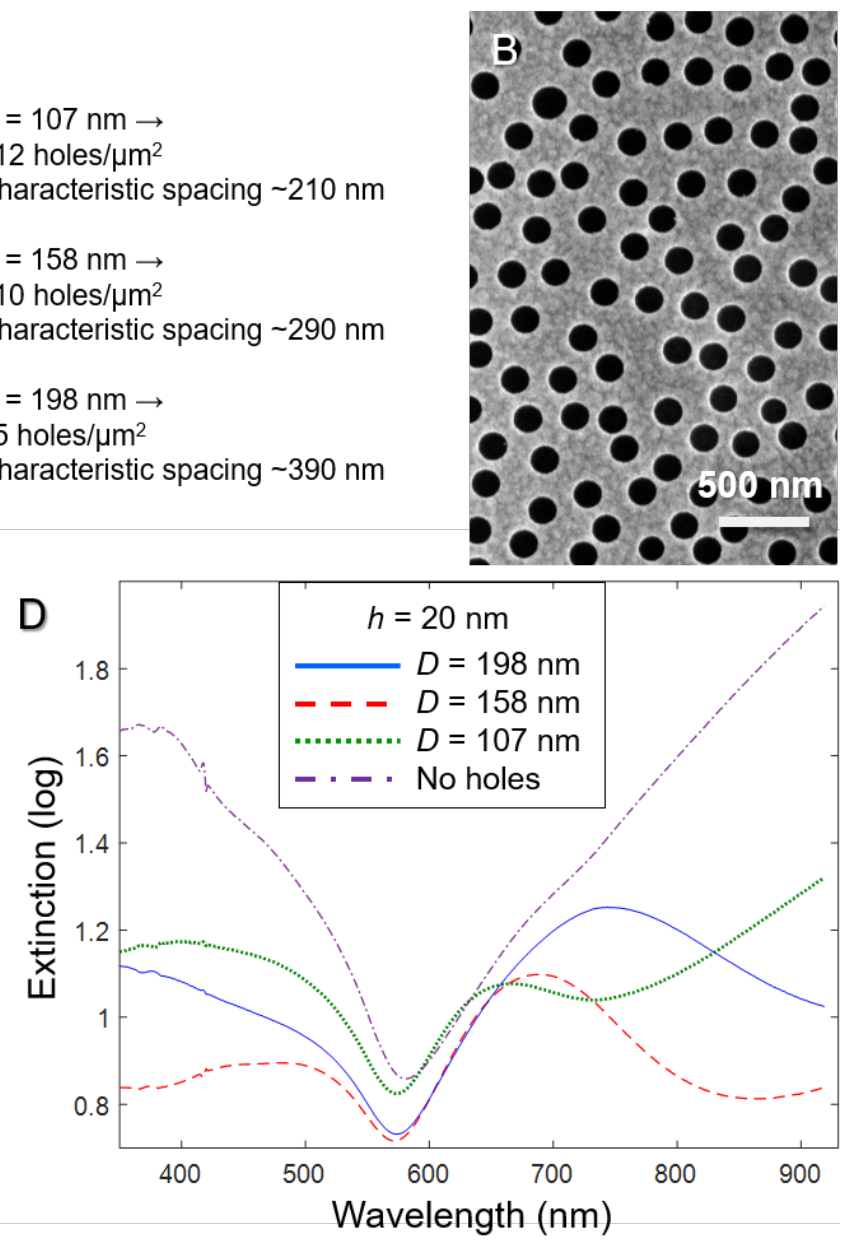

Figure 1. Characterizing the optical response of short-range ordered nanohole arrays in semitransparent $\mathrm{Cu}$ films. (A) Schematic of the structure and description of the different hole arrays. (B) Electron microscopy image taken from above of a sample prepared with $158 \mathrm{~nm}$ colloids. (C) Extinction spectra of samples prepared with a $30 \mathrm{~nm}$ Cu film. (D) Extinction spectra of samples prepared with $20 \mathrm{~nm} \mathrm{Cu}$.

We hypothesized that it should be possible to use the $\left(\mathrm{Al}_{2} \mathrm{O}_{3}\right.$ protected) $\mathrm{Cu}$ nanohole arrays with an $\mathrm{Al}$ mirror and a $\mathrm{Al}_{2} \mathrm{O}_{3}$ spacer to create MIN metasurfaces (Fig. 2A) with high chromaticity. As in our previous work with $\mathrm{Au}$ and $\mathrm{Ag}$ structures, ${ }^{11}$ we utilize a combination of Fabry-Perot interference (cavity mode) and surface plasmons to generate high resonant polarization-insensitive 
reflection. The highly reflective bottom $100 \mathrm{~nm} \mathrm{Al} \mathrm{mirror} \mathrm{has} \mathrm{essentially} \mathrm{zero} \mathrm{transmission}{ }^{30}$ and is covered by an $\mathrm{Al}_{2} \mathrm{O}_{3}$ film onto which the $\mathrm{Cu}$ nanohole arrays are prepared. By changing the $\mathrm{Al}_{2} \mathrm{O}_{3}$ layer thickness a wide color palette can be generated because the reflection from the cavity mode and the surface plasmon both change their individual resonance wavelengths due to coupling between the metal films. The plasmonic activity of the structure is vital since it causes resonant absorption and scattering of light, which strongly increases the color range compared to utilizing the cavity mode alone. This was obvious from the appearance of the same structures prepared without nanoholes in the $\mathrm{Cu}$ film, which showed weaker colors, especially for green and blue, regardless of $\mathrm{Al}_{2} \mathrm{O}_{3}$ thickness. By calculating the dispersion relation of surface plasmons in the MIN system using a generic method ${ }^{29}$ we confirmed plasmon coupling in the yellow to red region when the $\mathrm{Al}_{2} \mathrm{O}_{3}$ thickness lies in the interval 50-150 nm (Supporting Information). We also evaluated MIN structures prepared with only $\mathrm{Al}$ (or Ag) but those colors were much poorer (example in Supporting Information), especially in the red. This is to some extent because of the contribution from interband absorption in $\mathrm{Cu}(\mathrm{or} \mathrm{Au}),{ }^{24,}{ }^{28}$ but also because the plasmons are excited at higher energies in $\mathrm{Ag}$ and $\mathrm{Al}^{22}$ 
A
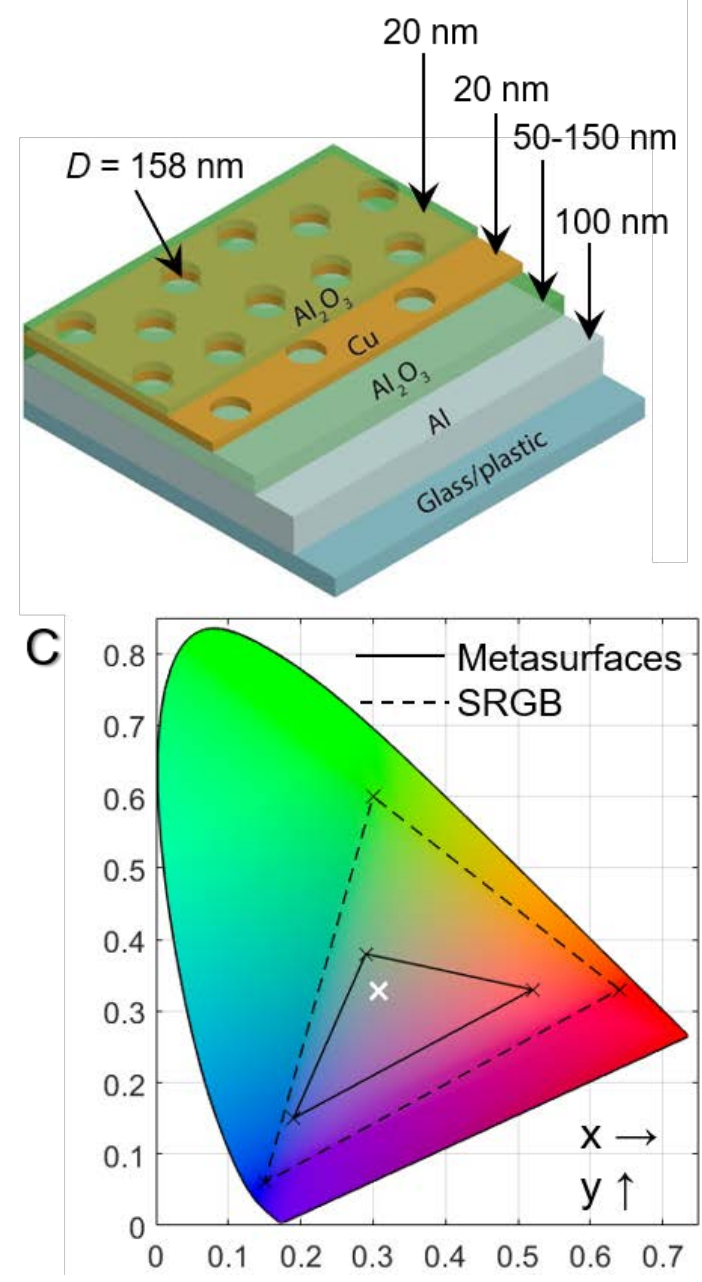
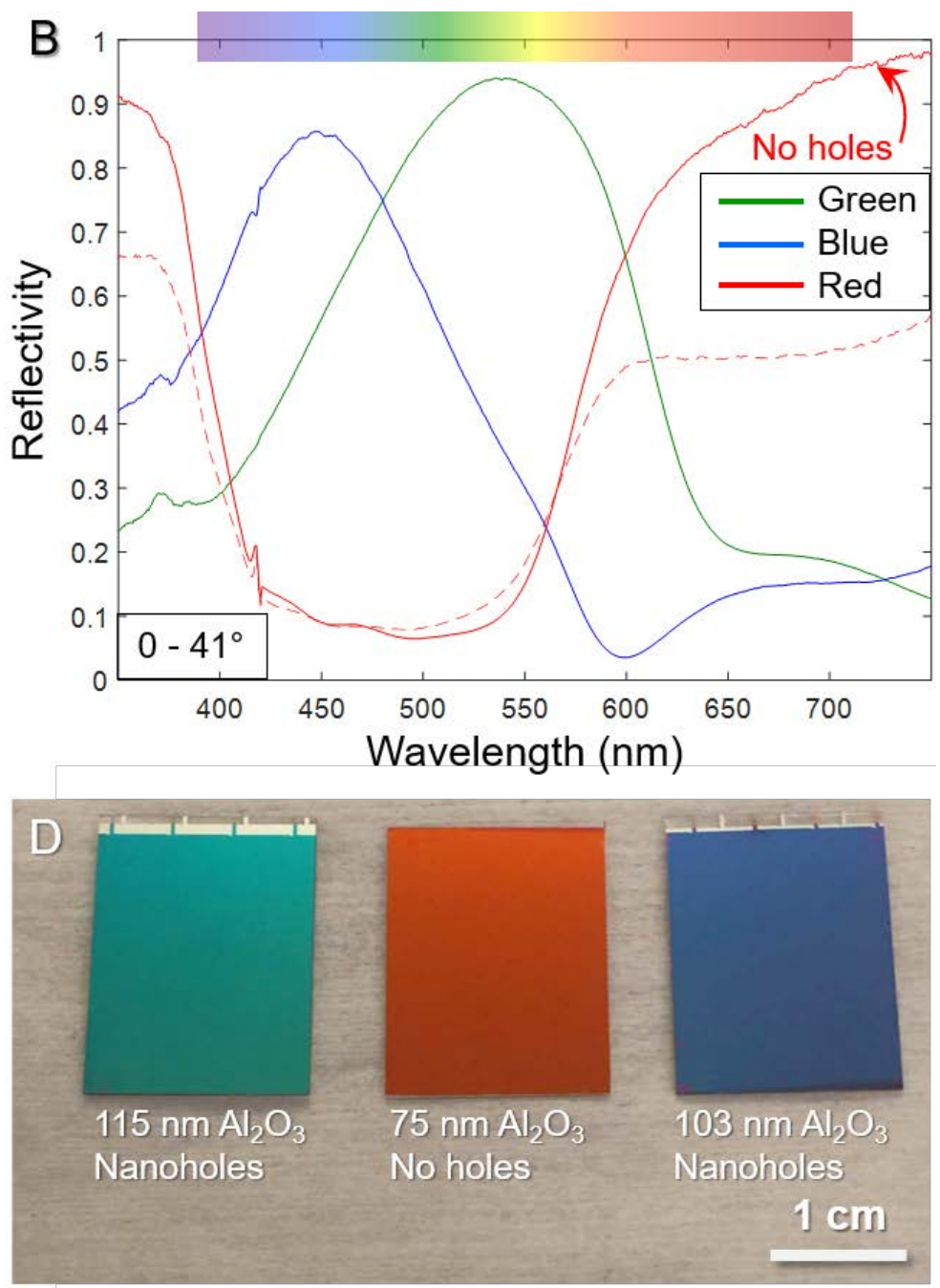

Figure 2. Reflective metal - insulator - nanohole array metasurfaces. (A) Layout of the structure with $\mathrm{Al}$ as bottom mirror, variable $\mathrm{Al}_{2} \mathrm{O}_{3}$ spacer and a short-range ordered array of $158 \mathrm{~nm}$ nanoholes in a $20 \mathrm{~nm}$ Cu film (see also Fig. 1C). (B) Reflectivity spectra in air with incident light ranging from zero to $41^{\circ}$ for the values of $\mathrm{Al}_{2} \mathrm{O}_{3}$ thickness that gives the best primaries. For red, there are no nanoholes in the $\mathrm{Cu}$ since this reduces the reflectivity (c.f. dashed spectrum). (C) CIE 1931 xy chromaticity space showing a comparison between the metasurfaces and standard RGB. (D) Photo of samples with the primary colors. 
In order to optimize the colors of the $\mathrm{Al} / \mathrm{Cu} \mathrm{MIN}$ structures in more detail, we tested several different nanohole arrays as top layer while also varying the $\mathrm{Al}_{2} \mathrm{O}_{3}$ thickness by creating a gradient on the surface. ${ }^{31}$ Concerning the nanohole array, we found that good colors were achieved for $D=$ $158 \mathrm{~nm}$ nanoholes in a $h=20 \mathrm{~nm}$ Cu film (spectra of MIN samples with other nanohole arrays in Supporting Information). In brief, this is because the resonance is reasonably strong and in the red region of the visible for $\mathrm{Cu}$ nanohole arrays of these dimensions. Even though the resonances should be sharper for $h=30 \mathrm{~nm}$ films (indicated in Fig. 1D), thicker films are not preferable since the reflectivity increases in the spectral regions outside the resonantly reflected color, which reduces the chromaticity. Concerning $\mathrm{Al}_{2} \mathrm{O}_{3}$ thickness, the best RGB primaries were achieved with approximately $75 \mathrm{~nm}$ for red, $103 \mathrm{~nm}$ for blue and $115 \mathrm{~nm}$ for green. (Note that these values are highly sensitive to the refractive index of the film, which may vary slightly with deposition technique etc.) The corresponding reflectivity spectra in air were measured at an incident angle interval from zero up to $41^{\circ}$ (Fig. 2B) using an air objective with NA 0.8. Importantly, despite this wide range of viewing angles the reflectivity is around $90 \%$ for each metasurface in the red, green or blue region respectively. The chromaticity (Fig. 2C) of the three primary colored metasurfaces was analyzed as described previously, ${ }^{11}$ showing that the gamut has the D65 white point included and covers $27 \%$ of the standard RGB. This so called CIE 1931 xy diagram is the established way of illustrating the chromaticity. Each color within the triangle is accessible by linear combinations (subpixels) of the coordinates of the primaries.

A photo of large-area metasurfaces representing the primaries is shown in Fig. 2D, illustrating the intense colors. (This photo, like other photos below, is taken with an ordinary mobile phone camera under normal lighting conditions with standard settings and the image has not been manipulated.) The red color was not improved by introducing nanoholes in the top film due to the 
surface plasmons excited in this spectral region, as confirmed by the reduced reflectivity when nanoholes are present in $\mathrm{Cu}$ (dashed spectrum in Fig. 2B), in agreement with previous results for Au nanohole arrays. ${ }^{32}$ Note that the reflectivity is reduced despite the wide angular interval in the measurement because the scattering occurs mainly at even higher angles (to the surface normal) for nanohole arrays of these dimensions. ${ }^{11}$ Therefore we did not use nanoholes for the red metasurfaces (and strictly speaking they are not plasmonic), but we emphasize again that the plasmonic nanoholes are necessary for achieving green and blue metasurfaces (as well as cyan and yellow), which are otherwise more magenta colored due to the increased reflectivity in the red. Numerical simulations of the reflectivity from MIN structures with literature values for permittivity ${ }^{28,} 30$ further confirmed the role of the surface plasmons (Supporting Information).

After optimizing the primary colors, we reproduced digital photos with the MIN metasurfaces by three standard photolithography steps (one each for patterning red, green and blue). Fig. 3A shows a "white standard" sample generated by RGB stripes, each $30 \mu \mathrm{m}$ in width. Upon switching to dark field illumination we could verify that the blue and green metasurfaces scatter yellow and red light respectively, confirming the plasmonic activity from the nanoholes in $\mathrm{Cu}$. As a control, there is no scattering detected from the red stripes since this is not possible for a thin film system without lateral structures. Color images were produced by translating each pixel in the source file into a RGB triplet with the relative intensities varied through the length of each subpixel stripe. Fig. 3B shows an example of such pixels $30 \mu \mathrm{m}$ wide and from zero to $100 \mu \mathrm{m}$ long. Several photos were successfully reproduced by the metasurface RGB pixels. (Some results cannot be shown here due to copyright issues.) In Fig. 3C we present a photo enabling a direct comparison between the pixelated plasmonic MIN structures and printing using a laserjet printer on ordinary paper. In both cases, the same image is reproduced, which is a colorful photo of the "Kuggen" 
building on our University campus in Gothenburg (source image included as Supporting Information). The metasurfaces clearly reproduce images very well in terms of colors. Importantly, this is despite the fact that for any other color than full white, only a fraction of the area contains metasurfaces. Still, the image clarity is obviously comparable to printed dyes on paper because of the high reflection. The image quality is further improved by the fact that both the cavity mode and the surface plasmon excitation are only weakly dependent on polarization ${ }^{33}$ and viewing angle (Supporting Information). The resolution is higher for the plasmonic image (282 dpi) and can be further improved since the limit is set by the optical lithography. 


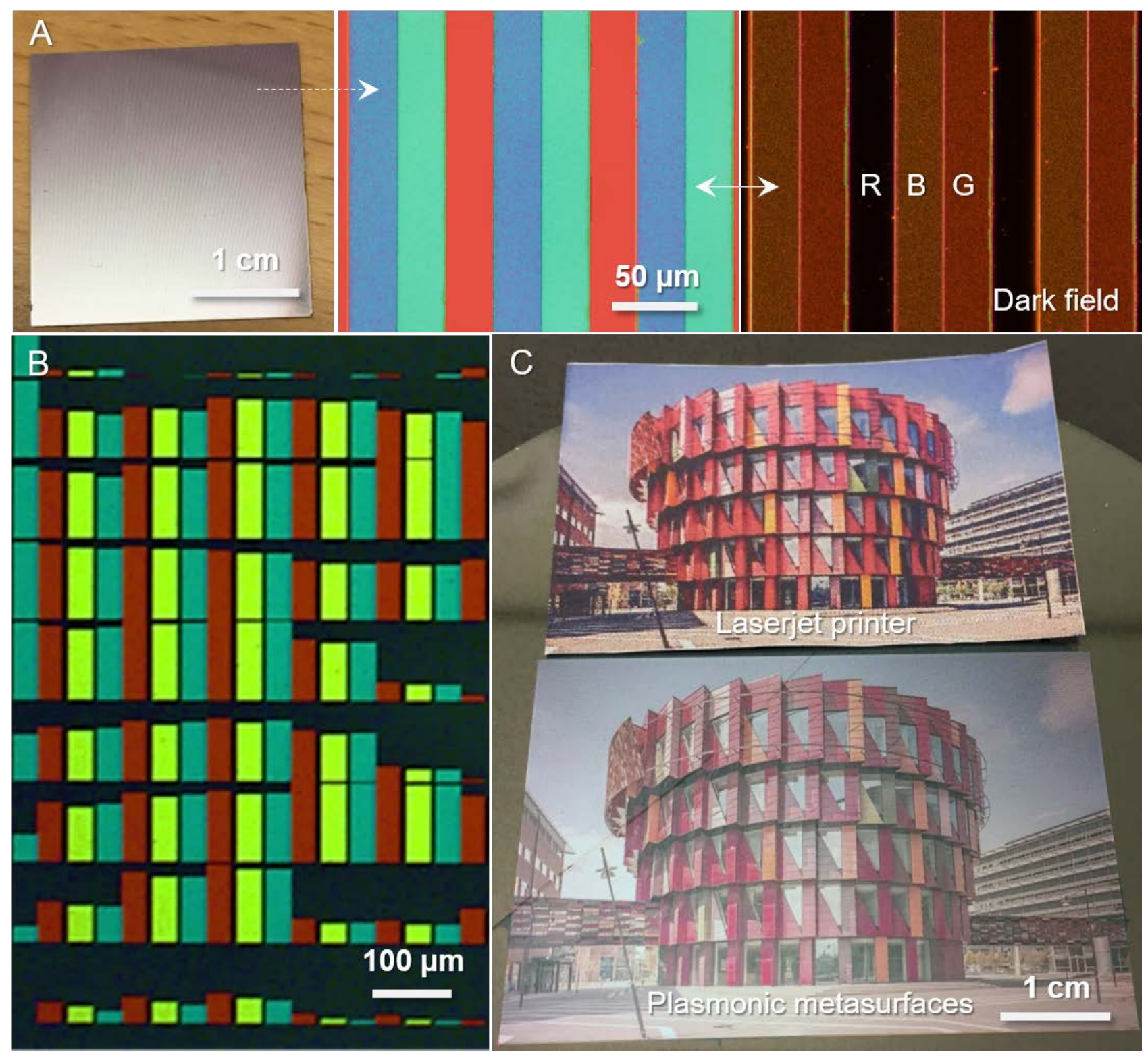

Figure 3. Reproducing color images by RGB pixel triplets. (A) RGB stripes $30 \mu \mathrm{m}$ in width generate a white sample. In dark-field illumination, scattering of the complementary colors is observed for green and blue metasurfaces, while the red structure without nanoholes gives no scattering. (B) Microscopy example image of metasurfaces patterned into RGB triplet with varying subpixel areas. (C) Photo providing a comparison between laserjet printing and plasmonic metasurfaces. The image reproduced shows the "Kuggen" building at the University campus. 
Original photo copyright belongs to Mabry Campbell Photography. Kind permission to use this photo has been obtained.

The most important step towards active plasmonic displays operating in reflection mode, i.e. electronic paper, is to implement electric modulation of the reflected intensity. Besides liquid crystals, this can be achieved with conductive ( $\pi$-conjugated) polymers that switch between light absorbing and transparent states, such as polypyrrole. ${ }^{34}$ Continuous $\mathrm{Au}$ structures or $\mathrm{Au}$ nanoparticles on a transparent conductor such as indium tin oxide (ITO) can remain stable while acting as one electrode to facilitate electrochromic switching of a conducting film on top of the metal. However, other metals like $\mathrm{Cu}$ are typically very unstable in electrolytes. ${ }^{26}$ Although the top $\mathrm{Al}_{2} \mathrm{O}_{3}$ layer of the MIN structures was sufficient to provide excellent stability in air for our metasurfaces, they were quickly destroyed when potentials were applied in an electrolyte. In order to make the structures compatible with electrochemistry we first deposited an additional insulating $\mathrm{SiO}_{2}$ coating of approximately $100 \mathrm{~nm}$. Next, to enable electrochromic switching, we used the highly conducting electrochromic polymer poly(3,4-ethylenedioxythiophene) (PEDOT). PEDOT is known to provide sufficient conductivity (also in its low-conductivity state) to facilitate its own switching without a supporting electrode. ${ }^{34,} 35$ It also provides a high and quite homogenous electrochromic contrast throughout the whole visible wavelength range, i.e. it essentially switches from black to transparent, albeit with a blue tone in the dark state. ${ }^{35,36}$ Thin films of PEDOT doped with polystyrene sulfonate (PEDOT:PSS) were screen-printed onto the metasurfaces, demonstrating compatibility with large-scale manufacturing. ${ }^{36}$

Electrochromic devices were finalized by immersing the polymer-modified metasurfaces in simple electrolytes and using ITO covered $(25 \mathrm{~nm})$ plastic as opposite window and counter electrode (Fig. 4A). The reflectivity changes upon switching the PEDOT ( $\pm 1 \mathrm{~V})$ in phosphate 
buffered saline (PBS) are shown in Fig. 4B (dashed vs full lines) for each primary colored metasurface. The reflectivity difference is $>0.4$ in the red region (for the red metasurface) and somewhat lower ( 0.3) for blue and green metasurfaces. By using plastic supports we could make electrochromic devices that were flexible and the radius of bending was limited simply by the thickness of the plastic support relative to the device area. Fig. 4C shows examples of devices and the color changes upon switching the voltage. Note that the PEDOT was always prepared directly on the MIN structures (separated only by the thin $\mathrm{SiO}_{2}$ film). This is important because if the "shutter" is a at a large distance from the colored surface proper image display in pixel arrays is problematic. This is a well-known limitation with LCD displays where the relatively thick liquid crystal layer ${ }^{4}$ required for good contrast restricts the viewing angle. In our active devices the absolute reflectivity in the "on" state (full lines in Fig. 4B) is reduced compared to the bare metasurfaces in air (Fig. 2B). This is in part because the PEDOT:PSS layer is not entirely transparent in the bright state, but also due to the electrochemical cell in the reflection spectroscopy setup. We emphasize that these results prove that electrochromism can be implemented on the nanostructures and although the optical contrast is sufficient for simple reflective displays ${ }^{36}$ there is clearly room for further optimization, for instance with respect to polymer thickness and composition $^{35}$ (discussion in Supporting Information). The thickness of the screen printed PEDOT:PSS films in this work was approximately $500 \mathrm{~nm}$ as measured by a profilometer (not shown). 

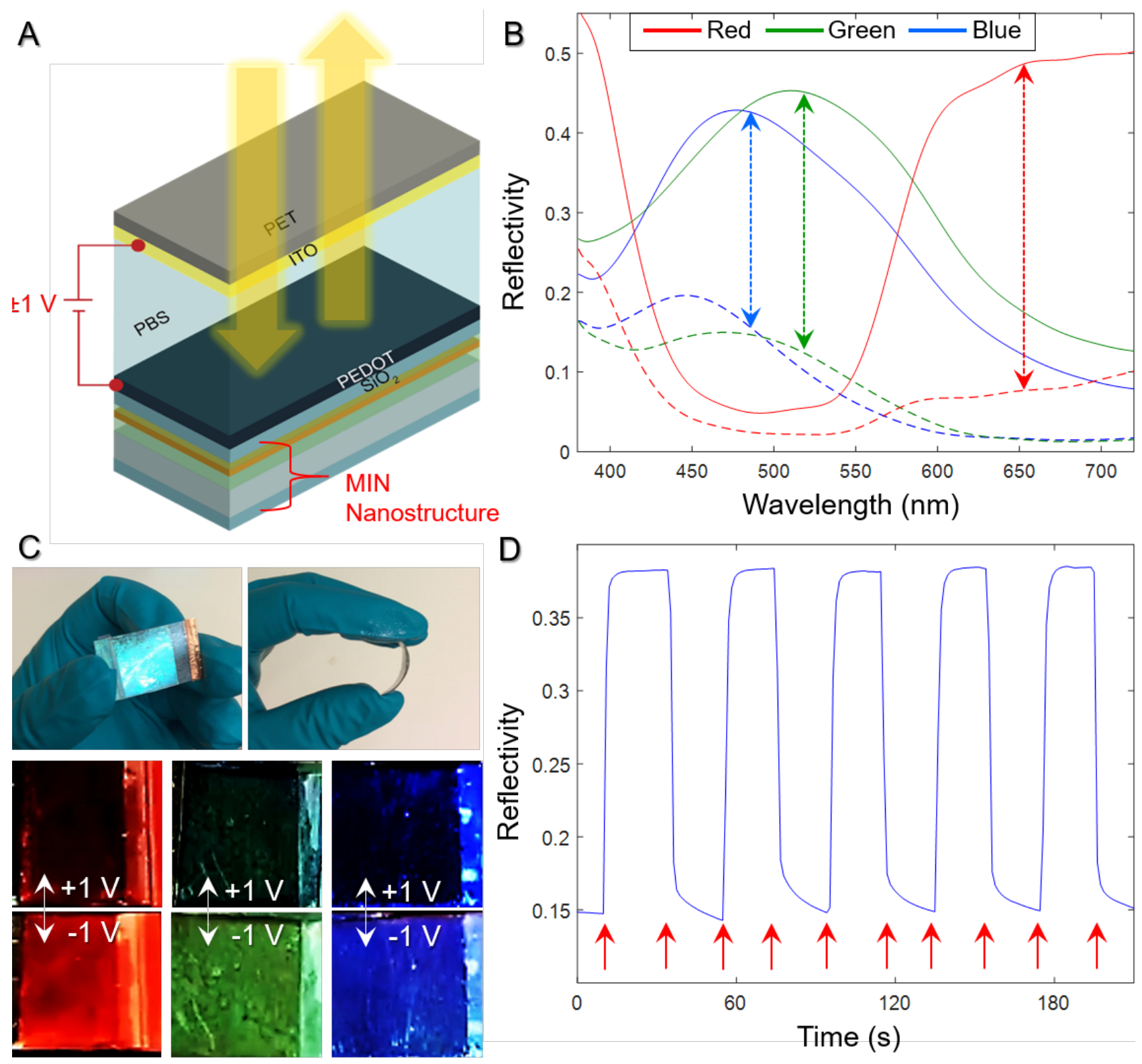

Figure 4. Electrochromic switching. (A) Cell design. (B) Reflectivity spectra of PEDOT-modified metasurface in the on and off states. (C) Pictures of a flexible device and electrochromic switching of the metasurfaces (approximately $2 \times 2 \mathrm{~cm}^{2}$ shown). (D) Switching dynamics (at $536 \mathrm{~nm}$ ) for a blue metasurface. The voltage is reversed between $+1 \mathrm{~V}$ and $-1 \mathrm{~V}$ as indicated by the arrows

Example of switching dynamics for a metasurface coated with PEDOT:PSS are shown in Fig. 4D (5 cycles). More than $90 \%$ of the reflectivity change occurs in less than $1 \mathrm{~s}$, which represents 
a response time suitable for electronic readers and simpler segmented reflective displays. The current trace gave a switching energy of $5.7 \mathrm{~mJ} / \mathrm{cm}^{2}$ (Supporting Information). Assuming an update every $\sim 10 \mathrm{~s}$ as typical for electronic readers, the power density for a display based on this technology is well in the ultralow region and even lower than electrophoretic ink. ${ }^{37}$ Further, PEDOT has good bistability or "optical memory”, ${ }^{34}$ i.e. the power consumption for maintaining an oxidation state (the same image in a reflective display) is extremely low. We refer to a video available as Supporting Information showing fast switching and bistability in a red device. The lifetime of the devices in terms of switching cycles can be very high since PEDOT can undergo hundreds of cycles depending on the environment. ${ }^{35}$ However, any defects in the $\mathrm{SiO}_{2}$ protection layer leading to contact between the nanostructure and the electrolyte would gradually destroy the samples.

In conclusion, we have shown that MIN metasurfaces combining $\mathrm{Cu}$ and $\mathrm{Al}$ can provide high chromaticity and intense reflection almost independent of polarization. Using RGB subpixels with varying area this enabled reproduction of color images of high quality over large areas using only abundant metals. Furthermore, the metasurfaces were made electrochromic by insulating the MIN structure with $\mathrm{SiO}_{2}$ followed by printing of PEDOT:PSS, which can be used for new types of reflective displays. The most challenging next step is to implement transistor arrays with the electrochromic switching ${ }^{36}$ in order to fully realize "plasmonic electronic paper” based on only abundant metals. The polymer composition and thickness can be further optimized for each primary color. There are also technical details that can be addressed to improve device stability and performance, such as increasing the charge capacity of the transparent counter electrode. ${ }^{38}$ Plasmonic electronic paper could fill a gap among existing display technologies by providing flexible reflective displays in color, thereby overcoming the limitations of electrophoretic ink (no 
colors) and reflective LCDs (low reflectivity and not bendable). At the same time other advantages are maintained, such as excellent visibility in direct sunlight, extremely low power consumption and even health benefits compared to emissive displays. ${ }^{39}$ 


\section{ASSOCIATED CONTENT}

\section{Supporting Information}

Experimental details. Reflectivity of metasurfaces with other $\mathrm{Cu}$ nanohole arrays. Simulated reflectivity of metasurfaces. Calculated dispersion relations. Data of current during PEDOT switching. Angle and polarization dependence. Photo of metasurfaces with only Al. Discussion on optimal polymer layer. The following files are available free of charge:

Image of the “Kuggen” building (Kuggen.tif)

Video showing switching and bistability of a red electrochromic device (bistability.avi)

\section{AUTHOR INFORMATION}

\section{Corresponding Author}

*adahlin@chalmers.se

\section{Author Contributions}

The manuscript was written through contributions of all authors. All authors have given approval to the final version of the manuscript.

\section{Funding Sources}

This work was financed by the Chalmers Nanoscience and Nanotechnology Area of Advance, the Swedish Foundation for Strategic Research (RMA11-0037), the Wenner-Gren Foundations, and the Swedish Government Strategic Research Area in Materials Science on Functional Materials at Linköping University (Faculty Grant SFO-Mat-LiU No 2009 00971). 


\section{Notes}

The authors declare no competing financial interest.

\section{ACKNOWLEDGMENT}

We acknowledge Prof. Mikael Käll (Chalmers) for useful discussions and Dr. Robert Brooke (Linköping) for initial experiments.

\section{ABBREVIATIONS}

MIN, metal insulator nanohole array; ITO, indium tin oxide; PEDOT poly(3,4-

ethylenedioxythiophene); RGB, red-green-blue; LCD, liquid crystal display; PBS, phosphate buffered saline.

\section{REFERENCES}

1. Freestone, I.; Meeks, N.; Sax, M.; Higgitt, C. Gold Bull. 2007, 40, 270-277.

2. $\quad$ Kristensen, A.; Yang, J. K. W.; Bozhevolnyi, S. I.; Link, S.; Nordlander, P.; Halas, N. J.; Mortensen, N. A. Nat. Rev. Mater. 2016, 2, 16088.

3. Gu, Y. H.; Zhang, L.; Yang, J. K. W.; Yeo, S. P.; Qiu, C. W. Nanoscale 2015, 7, 64096419.

4. Franklin, D.; Frank, R.; Wu, S.-T.; Chanda, D. Nat. Commun. 2017, 8, 15209.

5. $\quad$ Stec, G. J.; Lauchner, A.; Cui, Y.; Nordlander, P.; Halas, N. J. ACS Nano 2017, 11, 32543261.

6. Zhu, X.; Vannahme, C.; Hojlund-Nielsen, E.; Mortensen, N. A.; Kristensen, A. Nat. Nanotechnol. 2016, 11, 325-9. 
7. Chen, T.; Reinhard, B. M. Adv. Mater. 2016, 28, 3522-3527.

8. Kumar, K.; Duan, H.; Hegde, R. S.; Koh, S. C. W.; Wei, J. N.; Yang, J. K. W. Nat. Nanotechnol. 2012, 7, 557-561.

9. Duan, X.; Kamin, S.; Liu, N. Nat. Commun. 2017, 8, 14606.

10. Xu, T.; Walter, E. C.; Agrawal, A.; Bohn, C.; Velmurugan, J.; Zhu, W.; Lezec, H. J.; Talin, A. A. Nat. Commun. 2016, 7, 10479.

11. Xiong, K.; Emilsson, G.; Maziz, A.; Yang, X.; Shao, L.; Jager, E. W. H.; Dahlin, A. B. Adv. Mater. 2016, 28, 9956-9960.

12. Knight, M. W.; King, N. S.; Liu, L. F.; Everitt, H. O.; Nordlander, P.; Halas, N. J. ACS Nano 2014, 8, 834-840.

13. Olson, J.; Manjavacas, A.; Liu, L.; Chang, W.-S.; Foerster, B.; King, N. S.; Knight, M. W.; Nordlander, P.; Halas, N. J.; Link, S. P. Natl. Acad. Sci. USA 2014, 111, 14348-14353.

14. Olson, J.; Manjavacas, A.; Basu, T.; Huang, D.; Schlather, A. E.; Zheng, B.; Halas, N. J.; Nordlander, P.; Link, S. ACS Nano 2016, 10, 1108-1117.

15. Clausen, J. S.; Hojlund-Nielsen, E.; Christiansen, A. B.; Yazdi, S.; Grajower, M.; Taha, H.; Levy, U.; Kristensen, A.; Mortensen, N. A. Nano Lett. 2014, 14, 4499-4504.

16. James, T. D.; Mulvaney, P.; Roberts, A. Nano Lett. 2016, 16, 3817-3823.

17. Tan, S. J.; Zhang, L.; Zhu, D.; Goh, X. M.; Wang, Y. M.; Kumar, K.; Qiu, C.-W.; Yang, J. K. W. Nano Lett. 2014, 14, 4023-4029. 
18. King, N. S.; Liu, L. F.; Yang, X.; Cerjan, B.; Everitt, H. O.; Nordlander, P.; Halas, N. J. ACS Nano 2015, 9, 10628-10636.

19. Guay, J. M.; Lesina, A. C.; Cote, G.; Charron, M.; Poitras, D.; Ramunno, L.; Berini, P.; Weck, A. Nat. Commun. 2017, 8, 16095.

20. Sannomiya, T.; Scholder, O.; Jefimovs, K.; Hafner, C.; Dahlin, A. B. Small 2011, 7, 16531663.

21. Prikulis, J.; Hanarp, P.; Olofsson, L.; Sutherland, D.; Kall, M. Nano Lett. 2004, 4, 10031007.

22. Schwind, M.; Kasemo, B.; Zoric, I. Nano Lett. 2013, 13, 1743-1750.

23. Xiong, K.; Emilsson, G.; Dahlin, A. B. Analyst 2016, 141, 3803-3810.

24. McPeak, K. M.; Jayanti, S. V.; Kress, S. J. P.; Meyer, S.; Iotti, S.; Rossinelli, A.; Norris, D. J. ACS Photonics 2015, 2, 326-333.

25. Chan, G. H.; Zhao, J.; Hicks, E. M.; Schatz, G. C.; Van Duyne, R. P. Nano Lett. 2007, 7, 1947-1952.

26. Susman, M. D.; Feldman, Y.; Vaskevich, A.; Rubinstein, I. Chem. Mater. 2012, 24, 25012508.

27. Anema, J. R.; Brolo, A. G.; Marthandam, P.; Gordon, R. J. Phys. Chem. C 2008, 112, 17051-17055.

28. Johnson, P. B.; Christy, R. W. Phys. Rev. B 1972, 6, 4370-4379. 
29. Dahlin, A. B.; Mapar, M.; Xiong, K. L.; Mazzotta, F.; Hook, F.; Sannomiya, T. Adv. Opt. Mater. 2014, 2, 556-564.

30. Rakic, A. D. Appl. Optics 1995, 34, 4755-4767.

31. Ogier, R.; Shao, L.; Svedendahl, M.; Kall, M. Adv. Mater. 2016, 28, 4658-4564.

32. Tordera, D.; Zhao, D.; Volkov, A. V.; Crispin, X.; Jonsson, M. P. Nano Lett. 2017, 17, 3145-3151.

33. Reilly, T. H.; Tenent, R. C.; Barnes, T. M.; Rowlen, K. L.; van de Lagemaat, J. ACS Nano 2010, 4, 615-624.

34. Brooke, R.; Mitraka, E.; Sardar, S.; Sandberg, M.; Sawatdee, A.; Berggren, M.; Crispin, X.; Jonsson, M. P. J Mater. Chem. C 2017, 5, 5824-5830.

35. Malti, A.; Brooke, R.; Liu, X.; Zhao, D.; Andersson Ersman, P.; Fahlman, M.; Jonsson, M. P.; Berggren, M.; Crispin, X. J Mater. Chem. C 2016, 4, 9680-9686.

36. Ersman, P. A.; Kawahara, J.; Berggren, M. Org. Electron. 2013, 14, 3371-3378.

37. Fernandez, M. R.; Casanova, E. Z.; Alonso, I. G. Sustainability 2015, 7, 10854-10875.

38. Invernale, M. A.; Seshadri, V.; Mamangun, D. M. D.; Ding, Y.; Filloramo, J.; Sotzing, G. A. Chem. Mater. 2009, 21, 3332-3336.

39. Chang, A. M.; Aeschbach, D.; Duffy, J. F.; Czeisler, C. A. P. Natl. Acad. Sci. USA 2015, $112,1232-1237$. 